\title{
Pluripotent stem cells for pathological modelling of Hutchinson-Gilford Progeria Syndrome (HGPS) and drug discovery
}

Xavier Nissan

From 1st French-Italian meeting on laminopathies and other nuclear envelope-related diseases

Marseille, France. 15-16 January 2015

Progeria, also known as HGPS, is a rare, fatal genetic disease characterized by an appearance of accelerated aging in children. This syndrome is typically caused by mutations in codon 608 (p.G608G) of the LMNA leading to the production of a mutated form of Lamin A precursor called progerin. In HGPS, progerin accumulates in cells causing progressive molecular defects including nuclear shape abnormalities, chromatin disorganization, DNA damages and delay in cell proliferation. Although two clinical trials have recently produced promising results, as well as in vitro and in vivo, there is currently no cure for HGPS patients. In collaboration with the teams of Dr Nicolas Lévy (UMR_S 910) and Dr Lino Ferreira (University of Coimbra), we have addressed this challenge by developing two high throughput screenings using the unique self-renewal and pluripotency properties induced pluripotent stem cells (iPS cells). Accordingly, these studies revealed the potential therapeutic effect of two new classes of compounds rescuing both nuclear shape abnormalities and defects of differentiation through on one hand, an inhibition of the prenylation process and on the other hand, a decrease of progerin expression.

Published: 11 November 2015

doi:10.1186/1750-1172-10-S2-02

Cite this article as: Nissan: Pluripotent stem cells for pathological modelling of Hutchinson-Gilford Progeria Syndrome (HGPS) and drug discovery. Orphanet Journal of Rare Diseases 2015 10(Suppl 2):O2.

Correspondence: xnissan@istem.fr

CECS, I-STEM, AFM, Institute for Stem cell Therapy and Exploration of Monogenic diseases, Evry, France 